\title{
Factores bióticos y abióticos que determinan el asentamiento del meroplancton

\section{Resumen}

El meroplancton está constituido por organismos cuya fase larval se desarrolla dentro del plancton. En la presente nota de investigación se revisan los factores bióticos y abióticos que influyen en el asentamiento de los organismos meropláncticos, previo a su metamorfosis. Se contrastan los argumentos de las hipótesis embriologista y ambientalista, que son las posturas más aceptadas entre la comunidad científica.

Palabras | asentamiento, hipótesis ambientalista, hipótesis embriologista, CLAVE larvas, plancton.

*Universidad de Quintana Roo/margutierrez@uqroo.mx 


\section{Introducción}

Entre los seres vivos existen dos formas principales de desarrollo: el directo, cuando nace o eclosiona una versión en miniatura del adulto (como ocurre en los mamíferos), y el indirecto, cuando nacen o eclosionan uno o más estados larvales de vida libre y sufren metamorfosis, hasta que alcanzan la forma del estado adulto. Uno de los ejemplos más reconocidos de desarrollo indirecto es la metamorfosis de una oruga en una mariposa adulta.

Además de los insectos numerosos grupos, particularmente acuáticos, tienen un ciclo de vida complejo con varios estados larvales antes de desarrollarse como adultos. Estos grupos son importantes ecológica o económicamente, ejemplo de ellos son los crustáceos, equinodermos, cnidarios, esponjas y moluscos.

Las larvas, por definición, son morfológicamente distintas a sus formas adultas; con frecuencia, también sus nichos ecológicos y su nutrición son diferentes y se les ha clasificado en función de esos nichos. Por ejemplo, Chia (1974: I22) propuso una clasificación larval basada en su nutrición:

a) Lecitotróficas: con capacidad de dispersión corta, obtienen nutrimentos de la yema y pueden tener o no tubo alimenticio, el cual no es funcional. Se encuentran en la mayoría de mares polares y profundos. Su fase larval tiene una duración de dos a seis semanas.

b) Facultativas: con tubo digestivo; pueden alimentarse o no antes de la metamorfosis.

c) Planctotróficas: con capacidad de dispersión amplia, pueden alimentarse de detritus, plancton o ser parasíticas o adelfofígicas, presentan una enorme variedad de longevidad, incluso intraespecíficamente.

Por su tipo de hábitat las larvas se clasifican en pelágicas, bénticas, parasíticas o vivíparas (Chia, 1974: I2I; Scheltema, 1986: 290). Existen también larvas teleplánicas pelágicas, especialistas en recorrer grandes distancias y de larga duración (tres a seis meses) o capaces de retrasar el asentamiento (Scheltema, 1986: 290).

La fase larval está sometida a ciertas presiones ambientales que determinan su sobrevivencia. Estudios experimentales han mostrado que la temperatura, la calidad y cantidad de comida influyen en su desarrollo; de hecho, la disponibilidad del tipo de comida para cada estadio es de fundamental importancia (D'Asaro, 1975: 362; Scheltema, 1986: 30 I). 
La sobrevivencia larval también esta determinada por la tasa de depredación, sin embargo es difícil modelarla porque no es constante entre estadios particulares: diferentes depredadores prefieren comer distintos estados de desarrollo y, además, hay diferencias en los mecanismos de depredación (Scheltema, 1986:320; Pennington et al., 1986:239). Se ha establecido que el grado de depredación se relaciona con dos cosas, el tamaño del huevo y la longitud de la vida: entre más grande el huevo, menor riesgo de depredación, y entre más larga la vida, mayor riesgo (Chia, 1974: I27). También se ha encontrado que la depredación se relaciona con el tamaño de la larva y la profundidad que ocupa; así, las larvas pequeñas y en posición más superficial tienen mayor probabilidad de sobrevivencia que las larvas más grandes y colocadas en las regiones del fondo (Dekshenieks et al., 1997: 1596).

\section{Factores que influyen en el asentamiento larval}

La vida larval se divide por conveniencia en precompetente y competente. La primera etapa es un tiempo de desarrollo morfológico y fisiológico rápido; en la segunda, la larva se asienta y metamorfosea (Cameron, 1986: I 46; Scheltema, 1986:292). La duración del estado competente es variable (y puede alargarse) porque depende de que la larva encuentre un sitio adecuado para asentarse, pero el grado máximo al que se retarda la metamorfosis se alcanza cuando la larva deja de alimentarse o encuentra una señal adecuada para metamorfosear (Scheltema, 1986: 294; Davis \& Stoner, 1992: 3).

El retardo de la metamorfosis puede tener influencia en la biología y ecología larval; por ejemplo, en los equinodermos Dendraster excentricus y Echinarachnius parma los juveniles que metamorfosean muy pronto, después de que llegan a ser competentes, tienen tasas de crecimiento más altas que los juveniles con etapa de competencia más larga (Highsmith y Emlet, 1986: 359). La duración del retardo de la metamorfosis tiene una correlación significativa negativa con la temperatura (Lutz y Jablonski, 1978: 5I).

La metamorfosis se define como un cambio morfológico y fisiológico en el desarrollo (Davis y Stoner, 1992: I), que prepara a una larva pelágica para cambiar a una vida béntica, puede ser abrupto o gradual (Lutz, I978: 5 I; Cameron, 1986: 145) y definitivamente no ocurre en cualquier sitio; por lo tanto surge la pregunta: ¿Por qué las larvas se asientan donde lo hacen? La 
respuesta no es sencilla y hasta hoy se han definido dos posturas: la primera trata el problema desde un punto de vista ecológico, enfocándose en el comportamiento larval influenciado por la luz, la gravedad (Cameron, 1986: I45), las características del sustrato (Keshner y Lodge, 1990:319) y las corrientes (Eckman, 1983: 24I; Hill, I99I: 485); la segunda postura se ha concentrado en la capacidad de la larva para seleccionar un sustrato en particular, en respuesta a señales ambientales positivas o negativas (particularmente químicas); Hadfield (1986: 4I9) nombra a la primera postura como ambientalista y a la segunda embriologista.

Los trabajos que han tratado el problema desde el punto de vista ambientalista señalan la necesidad de ampliar el conocimiento de los factores asociados a la dispersión de las larvas, como la difusión de remolinos (dispersión azarosa de partículas como resultado de un flujo turbulento, considerando la advección), advección horizontal por corrientes (información de circulación estuarina y costera), transporte por corrientes oceánicas (como enfoque a las larvas teleplánicas) y un adecuado estudio del comportamiento larval, particularmente los cambios ontogénicos y su comportamiento asociado (Scheltema, 1986: 290).

Las larvas no pueden contrarrestar las corrientes y podría pensarse que su permanencia en zonas estuarinas responde exclusivamente a factores físicos que gobiernan el movimiento de partículas (Cameron, 1986: I 60; Roughgarden et al., 1988: | 460), pero también a la longitud de su fase planctónica larval (Sulkin y van Heukelem, 1986:269). De esta manera se ha explicado que exista poco asentamiento en lugares con espacio suficiente y una baja tasa de depredación: si las larvas entran en una corriente que se aleje de la costa por efecto de los vientos, no podrán asentarse; mientras que si ocurre lo contario habrá un asentamiento exitoso (Roughgarden, I988: I463;Alexander \& Rouhgarden, 1996: 273).

Un efecto similar lo tendrían corrientes que viajan en dirección perpendicular a la costa y que pueden ser un medio de transporte para organismos; esta postura asume que el mecanismo que transporta a las aguas frías que llegan a la costa es el mismo que transporta a las larvas. Este tipo de corrientes se conocen como ondas internas (corrientes de baja temperatura y velocidad que viajan entre capas de diferente densidad) (Pineda, I991:550).

Al suponer que el asentamiento larval depende exclusivamente de las corrientes, se pasan por alto tanto las variaciones de dirección y velocidad de 
circulación como las biológicas: con la ontogenia hay cambios rítmicos en la distribución vertical y la larva se puede colocar en ciertas corrientes que la mueven en diferentes direcciones y a distintas velocidades, por diferentes periodos (Sulkin y van Heukelem, 1986:276). Asimismo, las larvas responden a la gravedad y a la luz; dependiendo de sus necesidades, usan mecanismos como cilios o músculos, que producen diferencias en su distribución vertical (Cameron, 1986: I59) o lípidos almacenados y estructuras parachoque que les permitan controlar la velocidad de sedimentación (Chia et al., 1984: 1220).

Por otro lado, se ha observado que las larvas pueden alcanzar una velocidad promedio suficiente $(60 \mathrm{~cm} / \mathrm{min})$ para moverse en la columna de agua, controlando su posición vertical (Mileikovsky, I 973: I I). El periodo de migración vertical de las larvas está sincronizado con el periodo dial, y si consideramos que las larvas son capaces de controlar su posición vertical, entonces resulta un mecanismo de transporte eficiente, conocido como transporte de corriente selectiva dial. Si la larva usa ese mecanismo, será capaz de penetrar a los sitios de reclutamiento (Hill, I99I: 490).

Por otro lado, a nivel experimental, algunas larvas tuvieron preferencia por cierto tipo de sustrato; larvas de gastrópodos, por ejemplo, prefieren asentarse en sitios que semejen macrófitas, lo que sugiere que la relación entre las macrófitas y la densidad de invertebrados pueda estar influenciada por la morfología del alga y el comportamiento del invertebrado (Kershner y Lodge, 1990:5I), probablemente la preferencia por cierto tipo de alga esté relacionada con la disponibilidad de comida o el refugio que ofrezca contra la depredación (Crisp, 1974: 179). En general, las larvas de invertebrados prefieren asentarse en superficies rugosas, lo cual puede estar mediado por el tacto, más que por alguna señal química (Crisp, 1974: 180).Aunque la heterogeneidad del sustrato no tenga relación con la diversidad, sí tiene influencia sobre el porcentaje de cobertura larval, pudiera ser por efectos de hidrodinámica local o elección de la larva (Bourget et al., 1994: 50).

Ahora, desde un punto de vista embriologista, se asume que las larvas tienen un sistema nervioso que, al menos, traslada señales ambientales. Bajo este supuesto, la larva es capaz de seleccionar un sustrato particular para asentarse y metamorfosear (Hadfield, 1986:418) porque tienen capacidad de inspección (Crisp, 1974: I78). Este tipo de comportamiento ha sido estudiado a nivel experimental, los patrones observados son: 
a) Asentamiento gregario. Ocurre en respuesta a una señal inductora relacionada con los adultos (Woodin, 1986: 403). Es probable que las larvas detecten un compuesto volátil o soluble en el agua, que pudiera actuar como una feromona; aunque no hay una identificación precisa de los químicos involucrados (Burke, 1986: 329), hasta ahora ha sido posible extraer y caracterizar algunas moléculas que supuestamente inducen a la metamorfosis, como péptidos, aminoácidos y moléculas solubles al agua; pero, en general se desconocen los compuestos específicos involucrados y su estructura (Hadfield, 1986: 423).

b) Señales positivas. Es similar al anterior en el sentido de que las larvas detectan algún tipo de señal química que induce a la metamorfosis; este comportamiento puede explicar las relaciones especie-específicas entre larvas y material vegetal, como algas (Crisp, 1974:263) y macrófitas (Wallace y Edmonson 1986:315).

c) Respuesta a señales negativas. Algunas larvas son capaces incluso de evitar sitios de asentamiento donde se encuentre su depredador, si detectan la presencia su material de deshecho (Woodin, 1986: 405).

Los patrones anteriores tienen efecto sobre la aptitud: aparentemente, la asociación con coespecíficos, las relaciones especie-específicas y la respuesta a señales negativas, permiten a la larva disminuir la competencia, obtener alimento (Crisp, 1974: 177), refugio contra depredadores, incremento en la oportunidad de fusión en especies coloniales y reducción en la mortalidad juvenil (Wallace y Edmonson 1986: 321 ; Gotelli, 1990: 96).

Los seguidores del punto de vista embriologista no han podido responder qué tan especializada es la comunicación química entre las larvas y cuáles son los órganos involucrados en la secreción de los químicos. Por otra parte, las observaciones anteriores no se han probado en campo por la dificultad de detectar las moléculas químicas que intervienen.

\section{Conclusiones}

El asentamiento de una larva depende de la interacción entre factores complejos, tales como la velocidad de las corrientes, la posición de la larva en la 
columna vertical de agua, el comportamiento y el tipo de larva. Es muy importante la interacción de la tasa de asentamiento con la tasa de movimiento del agua, porque puede determinar el patrón espacial de asentamiento; sin embargo, también hay que considerar que éste puede depender de una señal química (positiva, como la presencia de coespecíficos, o negativa ante la presencia de un depredador) o de las características del fondo. Pero la probabilidad de que una larva se encuentre cerca del fondo depende de su posición en la columna de agua. El conocimiento de estos factores puede ser una herramienta útil para el manejo de organismos meropláncticos con importancia comercial o ecológica, para hacer inferencias en cuanto a distribución, abundancia o cobertura de las especies.

Agradecimientos

La autora agradece a Alberto de Jesús Navarrete, investigador El Colegio de la Frontera Sur-Chetumal, el material bibliográfico proporcionado para esta revisión; a Eduardo Suárez-Morales, Manuel Elías Gutiérrez (ambos de Ecosur-Chetumal) y a Adrián Cervantes Martínez (Universidad de Quintana Roo-Cozumel) por sus observaciones que mejoraron el contenido del trabajo.

\section{FUENTES CONSULTADAS}

Alexander, S. E. y J. Roughgarden (I996). "Larval transport and population dynamics of intertidal barnacles: a coupled benthic/oceanic model”. Ecological Monographs, vol. 66, 259-275.

Bourget, E. J. DeGuise y G. Daigle ( 1994$)$. "Scales of substratum heterogeneity, structural complexity and the early establishment of marine epibenthic community". Journal of Experimental Marine Biology and Ecology, vol. |8I, 3|-5|.

Burke, R. D. (1986). "Pheromones and gregarious settlement of marine invertebrate larvae". Bulletin of Marine Sciences, vol. 39, 323-33I.

Cameron,A. (1986). “Introduction to the invertebrate larval biology workshop: a brief background”. Bulletin of Marine Sciences, vol. 39, I45-I6I.

Chia, F.S. (1974).“Classification and adaptative significance of developmental patterns in marine invertebrates”. Thalassia Jugoslavica, vol. I0, I I I-I 30. 
J. Buckland-Nicks y Young C.M. ( 1984). “Locomotion of marine invertebrate larvae: areview”. Canadian Journal of Zoology, vol. 62, I2051222.

Crisp, D.J. (1974). "Factors influencing the settlement of marine invertebrate larvae”, en P.T. Grant y A.M. Mackie (eds.). Chemoreception in marine organisms. Academic Press, 177-265.

D'Asaro, Ch. (1965). “Organogenesis, development and metamorphosis in the queen conch, Strombus gigas, with notes on breeding habitats”. Bulletin of Marine Sciences, vol. I5, 359-4I6.

Davis, M. y A, Stoner (1992). Metamorphosis of queen conch larvae is triggered by trophic cues found in the nursery habitats. Technical report. Caribbean Marine Research Centre.

Deksheniecks, M. M., E.E. Hofmann,J.M. Klinck y E.N.Powell (1997).“A modeling study of the effects of size -and depth- dependent predation on larval survival". Journal of Plankton Research, vol. 19, I583-I 598.

Eckman, J. E. ( 1983). “Hydrodinamic processes affecting benthic recruitment”. Limnology and Oceanography, vol. 28, 24I-257.

Gotelli, N.J. (1990). “Stochastic models of gregarious larval settlement". Ophelia, vol. 32, 95- 108.

Hadfield, M. (1986). "Settlement and recruitment of marine invertebrates: a perspective and some proposals". Bulletin of Marine Sciences, vol. 39, 4I8-425.

Highsmith, R. C.y R. B. Emlet ( 1986)."Delayed metamorphosis: effect on growth and survival of juvenile sand dollars (Echinoidea: Clypeasteroidea)”. Bulletin of Marine Sciences, vol. 39, 347-36I.

Hill,A. E. (199I). “A mechanism for horizontal zooplancton transport by vertical migration in tidal currents". Mar. Biol., vol. III, 485-492.

Kershner, M. W. y D.M. Lodge (1990). "Effect of substrate architecture on aquatic gastropod-substrate associations”. J.N. Am. Benthol, Soc., vol. 9,319-326.

Lutz, R.A. y D. Jablonski (1978). “Larval bivalve shell morphometry: a new paleoclomatic tool?”. Science, vol. 202, 5 I-53.

Mileikovsky, S.A. (1973)." SSpeed of active movement of pelagic larvae of marine bottom invertebrates and their ability to regulate their vertical position”. Marine Biology, vol. 23, I I-I 7. 
Pennington, J.T., S.S. Rumrill y F. Shiang-Chia (1986). "Stage specific predation upon embryos and larvae of the pacific sand dollar Dendraster excentricus by II species of common zooplankton predators". Bulletin of Marine Sciences, vol. 39, 234-240.

Pineda, J. (199I)."Predictable upwelling and the shoreward transport of planktonic larvae by internal tidal bores”. Science, vol. 253, 548-55I.

Roughgarden, J., S. Guines, y H. Possingham (1988). "Recruitment dynamics in complex life cycles”. Science, vol. 24I, I 460-I466.

Scheltema, R. S. (1986). “On dispersal and planktonic larvae of benthic invertebrates: an eclectic overview and summary of problems". Bulletin of Marine Sciences, vol. 39, 290-322.

Sulkin, S. D.y F.Van Heukelem ( 1986). "Variability in the length of the megalopal stage and its consequence to dispersal and recruitment in the portunid crab Callinectes sapides Rathbun". Bulletin of Marine Sciences, vol. 39, 269-278.

Wallace, R. L.yW.T. Edmonson (1986)." Mechanism and adaptative significance of substrate selection by a sessile rotifer". Ecology, vol. 67, 3I4-323. Woodin, S.A. ( 1 986). “Settlements of infauna: larval choice?”. Bulletin of Marine Scienses, vol. 39, 40I-407. 
